\title{
International Union of Geological Sciences (IUGS) Ad Hoc Review of Initiative on Forensic Geology (IFG)
}

\author{
Jose P. Calvo \\ Secretary General, IUGS.E-mail: jpcalvo@ucm.es
}

An Ad Hoc Review Committee (ARC) was convened to consider the IUGS Initiative on Forensic Geology (FG). The meeting held on $4^{\text {th }}$ April 2016 at Piccadilly Place, Manchester, UK under the Chairmanship of Prof. Jose P. Calvo (JC), Secretary General, IUGS. The other attendees of the meeting were Prof. Marko Komac (MK), Vice-President of the IUGS, Dr. Laurence Donnelly (LD), Chair of the IUGS Initiative on Forensic Geology (IFG) and a Forensic Geologist and Police Search Adviser, Dr. Alastair Ruffel (AL), Forensic Geologist at Queens University, Belfast, who has significant experience in forensic geology and in working with law enforcement agencies, and Inspector Colin Hope (CH), National Search Advisor at the UK National Crime Agency.

Prof. Calvo welcomed the attendees, thanked Dr. Donnelly and Arup for facilitating the review meeting and described the purpose and terms of the ARC i.e. "As specified by the Statutes and Bylaws of the IUGS, the Executive Committee is required to undertake a formal review of all Commissions and other bodies funded by the Union on an approximate 4 year cycle. These reviews provide accountability for the expenditures incurred by the Union". Under IUGS Bylaws, the ARC panel must be made up of a minimum of two IUGS members (JC and MK) together with one external member who is preferably a subject matter expert $(\mathrm{CH})$.

LD gave a presentation that described the background, history, evolution, development, methodologies and achievements to date of the IFG. The 'recent' evolution of FG, traced back to 1994, when its application was considered in relation to ground searches for graves and other buried items, has since reached several milestones that include (i) presentation on FG to the All-Party Parliamentary Group for Earth Science, at Westminster Palace, House of Commons, in London, UK (2002) and (ii) establishment of (a) the Geological Society of London, Forensic Geoscience Group (FGG, 2002), (b) Working Group on FG as part of IUGS Geoscience for Environmental Management (GEM, 2006); (c) Geoforensic International Network (GIN, 2009) and (d) IFG as a funded initiative within the IUGS (2011).

The role of the IFG within the IUGS Science Policy is structured around three key themes:

- Outreach: Educating and informing police, law enforcement organisations, geologists, geoscientists and the 'general public' about forensic geology.

- Capacity Building: By raising levels of awareness, knowledge and providing information about forensic geology.

- Technology transfer and training: Through improvement in skill sets.

LD's presentation also highlighted the aims and objectives of IUGS-IFG, its overview of achievements during last five years, its plans for the coming years, the structure of FG committees, the Geoforensic International Network (GIN) and the benefits of IFG for IUGS.

The aims and objectives: of IUGS-IFG is to develop FG internationally and to promote its applications. The objectives are to (i) collate and disseminate data and information on FG as applied to policing and law enforcement, criminal, environmental and civil investigations; (ii) promote international meetings, seminars, conferences and training; (iii) develop a committee to act as principal advisers, collaborators and active participants; (iv) develop an international network where by each member will act as a principal contact in their respective country for the collation and dissemination of information on FG; (v) collate, make available and where appropriate review any existing documentation and publications in FG and (vi) produce a document endorsed by the Committee to be called; 'A Guide to Forensic Geology'.

Summary of achievements of IUGS-IFG during 2011-2016 an overview: The FG initiative was successful in forming professional international groups/association in 36 countries, conducting international events including those for school children, initiating new academic courses at graduate and post-graduate levels, promoting research (in many universities across the globe), publication of articles in police journals/magazines, and discussions in Westminster Palace, House of Commons. It developed collaborations with Police departments in UK (ground searches for burials), Russia, Abu Dhabi (with the Police, Navy and Coast Guard), Australia (followed by a training session with the Victoria Police), Brazil, Japan, Hong Kong, China and Canada.

Looking forward: The IUGS-IFG Looks forward during 20162017 to (i) development and modification of the IUGS-IFG web site; (ii) continuing to increase levels of support to police and law enforcement, whilst acknowledging that some investigative tactics must remain confidential and therefore cannot be publicised; (iii) produce supportive and informative documentation, protocols and guidance for law enforcement with regard to the application of forensic geology; (iv) a themed issue of 'Episode'; (v) participation at the $35^{\text {th }}$ IGC in Cape Town; (vi) production of "A Guide to Forensic Geology" and its translation (key points) into other languages (vii) outreach, capacity building, training and advanced training; (viii) development of FG in developing countries; (ix) expansion and diversification of FG in environmental forensics, geotechnical geoforensics, geohazards and fraudulent crimes (x) possible participation in TV and other media presentations regarding the inclusion of FG within a police 'cold case' and search; (xi) FG article in Geoscientist (GSL), December 2016 and (xii) planning for the 3rd Iberoamerican forensic geology course, Argentina (2017). In all instances, the support, contribution and marketing of IUGS will continue be referenced and the logo included on any materials published or publicised, as has been the case to date.

The structure of the IFG committee: The structure of the IFG committee and its regional representation was shown to include partners and stakeholders such as Police and Law Enforcement officials, business and commercial representatives and students, 
academics from a range of scientific disciplines and consultants. The IFG committee comprises members from: Arup (England); Commonwealth Scientific and Industrial Research Organisation (CSIRO) (Australia); The James Hutton Institute (Scotland); California Department of Justice (USA); Australia Federal Police (AFP) (formerly, UK Police National Search Adviser) (Australia); Federal Bureau of Investigation (FBI) (USA); SUNY Buffalo State (USA); Queens University Belfast (QUB) (Northern Ireland); Microtrace LLC (USA); Helford Geoscience LLP (UK); University College London; JDI Centre for the Forensic Sciences; Independent Consultant Forensic Geologists (Global); Washington State Patrol Crime Laboratory (USA); Instituto Nacional de Medicina Legal y Ciencias Forense, Bogota (Colombia); Brazilian Federal Police (BFP) (Brazil); Geoscienze Forensi Italia (formerly, Servizio Polizia Scientifica) (Italy); Russian Federal Centre of Forensic Science, Ministry of Justice, Moscow (Russia); Abu Dhabi Police, Forensic Geology Group, United Arab Emirates (UAE); National Research Institute of Police Science, Japan (Japan); University of Canberra (Australia); University of Technology, Sydney (Australia); Dalhousie University, Nova Scotia (Canada); and University of Pretoria (formerly, Forensic Science Lab, South African Police Service) (South Africa).

The Geoforensic International Network (GIN): The GIN brings together forensic geologists, geoscientists and police/law enforcement officers from around the world to develop and promote FG. It has 35 member countries and another 11 countries are targeted as potential members.

The benefits of IFG for IUGS: The main benefits of IFG for IUGS include global recognition as leading the development of FG, forging global collaboration, raised public, political, media and police awareness of FG, remain non-political, non-governmental, impartial, non-profit making, advancement in FG education and teaching, involvement in public affairs, advance global research, production of authoritative documents and guidance, standardisation and refinement of geological applications to forensic and police investigations. This is high profile exposure of IUGS that would enhance visibility of IUGS in new fields and to new audiences not previously reached by IUGS.

The ARC Comments: JC thanked LD for his informative presentation and the IFG was congratulated on their global achievements by all present. The concept of FG was now universally accepted as valid and worthy of development and further support. On a comment from MK as to whether the IUGS was referenced in outreach material produced and released by the IFG, LD confirmed that the support from the IUGS was recognised as being critical to the successful delivery of IFG functions and that all material and presentations refer to the IUGS and all materials include the IUGS logo. On another question from MK regarding the steps taken to increase the diversity in background of members, LD replied (i) a variety of outreach, knowledge transfer and training opportunities have been held for schoolchildren, support to BSc, MSc courses and $\mathrm{PhD}$ research projects (ii) presentations have frequently been delivered to a variety of audiences including academics, co-professional, consultants, police and law enforcement organisations, and (iii) work is ongoing to establish representation in the 'harder to reach' locations and developing countries. Contacts are currently being developed in, for example, Africa, Asia, Latin America, China and Hong Kong. The requirement to increase access to all aspects of FG for persons from all backgrounds is recognised and a considerable amount of work has been focussed on engagement with younger audiences, outside of the traditional 'professional' audience, together with the use of new technologies to increase accessibility.

$\mathrm{CH}$ enquired about the availability of professional accreditation to reassure law enforcement agencies that the geologist is a credible and experienced forensic asset. LD/AR replied that the IFG provides such reassurance through the establishment of a peer review network involving appropriately qualified and suitable experienced professionals with experience, gained through exposure to operational case work working with the police. The IFG network facilitates knowledge transfer and the development of emerging techniques and technologies', ensuring that support is provided by those who are informed, current and credible. IFG is addressing accreditation and regulation in $\mathrm{FG}$.

On a question regarding marketing of IFG by MK, LD replied that it is done through the website, flyers (in multiple languages), newsletters, peer reviews of journals, articles and books, reviewed academic papers and articles, attendance at events, guest lectures and keynote lectures, conferences and congresses. Every opportunity is taken to market the IFG through law enforcement CPD events. As stated above, the support of the IUGS is referenced throughout.

The ARC discussed future challenges, options and funding of IFG. The challenges identified are:

- The ongoing support of the IUGS is recognised as vital but additional funding sources are also being sought for continuation and expansion of the IFG.

- Competence and accreditation of professionals involved.

- Alignment with other forensic sciences and regulations.

- Personal security of members when working internationally.

- Confidentiality of recommendations and methodologies when applied within the law enforcement context.

- Excessive requests for publications.

- Maintenance of relationships with key individuals within law enforcement, as officer retention and tenure rules can often affect staff and their cyclic turn over.

- Management of the media and journalists.

- Protection of confidential techniques and methods in FG to reduce forensic awareness of criminals, terrorists and those involved in organised crime.

IFG accounts are submitted annually and are audited externally to ensure full transparency. A surplus of approximately $£ 9,600$ was reported in 2015 following the good management of accounts. However, a number of initiatives and engagement events are planned for 2016 that (whilst this surplus will be of benefit in supporting) will require additional funding if the IFG is to continue to expand and to further develop the awareness of FG. The accounts are available at all times for review and in the interest of transparency of IFG and IUGS members.

The options for the future of the IFG discussed were (1) to close the initiative; (2) to extend the initiative and enable the continuation and advancement of FG as per the outline at 2.6 above; (3) to revert to the Geological Society of London, Forensic Geoscience Group and/or (4) to establish an international association, group or society.

Discussion: Under the IUGS Articles 29 and 30, it was identified 
that there is no time limit set for the establishment or existence of IUGS Task Groups or Initiatives. The primary IUGS requirements are that an Initiative should continue to be relevant and have significant visibility within all applicable communities.

It was agreed that the IFG effectively and efficiently complies with these requirements. It was reiterated (MK) that the IFG is to be congratulated for its achievements to date and that all applicable targets have been, or are being, achieved.

Of the options for future progression identified above, option 2 was agreed by the review group as the most appropriate.

Opportunities exist for collaboration of IFG with new IUGS initiatives such as 'Human Activities and the Geological Environment', 'Geotechnics' and 'Medical Geology' were also thought to be particularly relevant. Contact details can be obtained from the Secretary General.

Funding within the IUGS remains limited in terms of availability and is subject to ongoing scrutiny. In 2016, due to the significant costs associated with holding a session at the $35^{\text {th }}$ IGC in Cape Town, the IUGS Executive Committee has approved a sum of 10,000 USD for 2016. Future funding for IFG will be determined on an annual basis by the IUGS Executive Committee.

Conclusion: During the period 2011-2016 IFG has met and exceeded its objectives. IUGS, via IFG, has provided the opportunity for the development and advancement of FG throughout the world resulting in this multidisciplinary science become firmly established in the geology profession (research \& practise), policing and law enforcement. Looking forward, FG requires continued support and development in targeted parts of the globe and developing countries. Its future priorities included documentation, protocols, alignment with forensic science, training, regulation and accreditation.

\section{Recommendations:}

- The ARC recommends the continuation and extension of the IFG.

- Funding for 2016 will be 10,000 USD.

- Future funding will be determined on an annual basis by the
IUGS Executive Committee based on the IFG performance and available funds in IUGS budget.

IFG should:

- continue to increase the diversity of membership of IFG and expand the committee.

- engage with new technologies, particularly those available for the deployment of geological applications 'on the ground'.

- increase the diversity of opportunity for all wishing to access FG, particularly young people and those from 'harder to reach' communities and developing nations.

- develop relationships and engage with other IUGS Task Groups and Initiatives.

- enhance accessibility to IFG materials through translation and distribution internationally.

- in the police and law enforcement context, consider the opportunities presented by including other relevant scientific disciplines, for example archaeology and ecology, in blended training delivery.

- promote engagement and compliance with established forensic standards such as those issued by the Forensic Regulator in the UK.

- publish 'A Guide to Forensic Geology'. IUGS is very supportive for the development and publication of this guidance and looks forward to examination of the final product. IFG will need to liaise with GSL and IUGS for clarification of protocol between two groups in relation to its publication.

- further develop the IFG website to enhance visibility.

- establish an 'excellence in research' framework and provide support through a peer review network.

Towards the end of the ARC Meeting, JC thanked all parties for their attendance and participation. The ARC acknowledges the exceptional efforts of Dr Laurance Donnelly and the achievements of the IFG. LD, on behalf of the IFG, thanked the IUGS Executive Committee, for continued support of the initiative and acknowledged the hard work, support and dedication of the IFG committee and Inspector Colin Hope. The meeting closed at 15:30 hrs. 